\title{
Synergistic bactericidal effect of low-frequency and low-intensity ultrasound combined with levofloxacin-loaded nanoparticles on M. smegmatis in macrophages
}

\section{Shuang Xie}

State Key Laboratory of Ultrasound in Medicine and Engineering $₫$ College of Biomedical Engineering $\mathbb{}$ Chongqing Medical University

\section{Gangjing Li}

State Key Laboratory of Ultrasound in Medicine and Engineering $₫$ College of Biomedical Engineering $\mathbb{}$ Chongqing Medical University

\section{Yuru Hou}

State Key Laboratory of Ultrasound in Medicine and Engineering $₫$ College of Biomedical Engineering $\rrbracket$ Chongqing Medical University

\section{Min Yang}

State Key Laboratory of Ultrasound in Medicine and Engineering $₫$ College of Biomedical Engineering $\rrbracket$ Chongqing Medical University

\section{Fahui Li}

State Key Laboratory of Ultrasound in Medicine and Engineering $₫$ College of Biomedical Engineering $\mathbb{}$ Chongqing Medical University

\section{Jianhu Li}

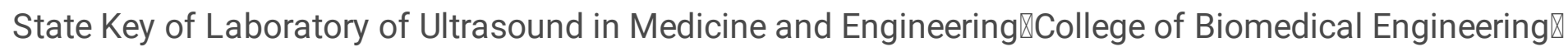
Chongqing Medical University

\section{Dairong Li}

Department of Respiratory and Critical Care Medicine区the First Affiliated Hospital of Chongqing Medical University

\section{Yonghong Du ( $\nabla$ duyonghong@cqmu.edu.cn )} https://orcid.org/0000-0002-6789-5596

\section{Research}

Keywords: levofloxacin-loaded nanoparticles; low-frequency and low-intensity ultrasound; bactericidal effect; M. smegmatis; macrophages

Posted Date: March 3rd, 2020 
DOI: https://doi.org/10.21203/rs.3.rs-15744/v1

License: (c) (1) This work is licensed under a Creative Commons Attribution 4.0 International License. Read Full License 


\section{Abstract}

Purpose Tuberculosis is a highly infectious disease caused by Mycobacterium tuberculosis (Mtb) which often parasites in macrophages. The present study was to investigate the bactericidal effect and underlying mechanisms of low-frequency and low-intensity ultrasound (LFLIU) combined with levofloxacin-loaded nanoparticles (LEV-NPs) on M. smegmatis (a surrogate of Mtb )in macrophages. Methods and results LEV-NPs were prepared by a double emulsification method. The characterization, such as average diameter, zeta potential, polydispersity index and morphology, and in-vitro drug release efficiency of LEV-NPs were investigated. M. smegmatis in macrophages was treated by LEV-NPs combined with $42 \mathrm{kHz}$ ultrasound irradiation at an intensity of $0.13 \mathrm{~W} / \mathrm{cm} 2$ for $10 \mathrm{~min}$. The results showed ultrasound could significantly promote phagocytosis of nanoparticles by macrophages ( $p$ $<0.05)$, further ultrasound combined with LEV-NPs could promote the production of macrophage ROS, and the apoptosis rate of macrophages was significantly higher than that of the control $(p<0.05)$. Transmission electronic microscope showed M. smegmatis cell wall was ruptured, the cell structure was incomplete, and the bacteria received severe damage in the ultrasound combined with the LEV-NPs group. Activity assays showed that ultrasound combined with LEV-NPs exhibited 10-fold higher antibacterial activity against $\mathrm{M}$. smegmatis residing inside macrophages compared with free drug. Conclusion Our data demonstrate that ultrasound combined with LEV-NPs have a great potential to therapy of tuberculosis.

\section{Introduction}

Tuberculosis (TB) is an infectious disease, which is the main cause of ill health. It is one of the top ten causes of death in the world, and also the number one killer of a single infectious disease (ranking above HIV/AIDS). The pathogenic bacterium of TB is Mycobacterium tuberculosis (Mtb) can be spread when people who are sick with TB expel bacteria into the air, for example, by coughing. The typical manifestation of tuberculosis is pulmonary tuberculosis, but it can also be seen in other sites as extrapulmonary tuberculosis[1-3]. According to the World Health Organization, 1.4 million people died of tuberculosis, and 10 million new cases were reported in 2019[4]. Mtb, an intracellular bacterial pathogen, is characterized by a thick cell wall, poor permeability, which makes it difficult for drugs to diffuse into Mtb. Systemic chemotherapy is currently available for TB treatment[5]. Compared with other bacterial infections, the treatment period of tuberculosis is prolonged, which requires more than six months of treatment. This long, intensive, and high systemic exposure with more side effects is one of the main causes of poor patient compliance or nonadherence with prescribed treatments, which leads to the significant emergence of drug-resistant strains. The treatment of multidrug-resistant tuberculosis patients requires more expensive and toxic drugs with poor outcomes[6, 7].

The intracellular survival of Mtb itself in macrophages plays a central role in the pathogenesis of TB, which limited the bioavailability of the dosed antibiotics in the target area. One of the recent developments in antibacterial strategies in addressing these challenges lies in exploring antimicrobial nanoparticles and antibiotics delivery systems as new tools to tackle the current challenges for efficient 
antibiotics delivery and reducing drug toxicity[8-10]. Tuberculosis patients will benefit from the development of new tuberculosis drugs, treatment regimens, and treatment modalities.

The application of nanoparticles as drug and bioactive active molecular carriers has shown attractive potential in disease treatment applications during the last few years to achieve controlled release of drugs. Several types of nanoparticles made of various polymers have been designed with the use of drug encapsulation for targeting pathogenic bacteria[11-13]. Among these materials, poly(lactic-co-glycolic acid) (PLGA) is Food and Drug Administration approved for human use, and PLGA particles are the most widely-applied type of particles due to their biocompatibility and biodegradability. The extensive use of PLGA nanoparticle-based drug delivery systems is promising in the field of antimicrobial infection, with higher efficiency and less adverse effects[14].

Low-frequency and low-intensity ultrasound (LFLIU) is a novel and noninvasive method to reversible, selective, and safe application of chemotherapy drug delivery $[15,16]$. The ultrasound-induced increase in drug penetration into cells is believed to result from oscillations in gas bubbles in media[17]. These oscillations cause cavitations and disruptions close to the cell surface that shear membrane, making them more permeable to small molecules, thus allows increased drug diffusion[18]. Recent reports showed that LFLIU had been widely adopted by medical researchers to improve the bactericidal effect of antibiotics against planktonic bacteria, bacterial biofilms, chlamydia, ect. in vitro and in vivo[19-21]. Numerous studies in the field of ultrasound-mediated intracellular delivery of drug have demonstrated that the application of ultrasound has an improved efficacy of either free drugs, antibiotics encapsulated in nanoparticles. Our previous research shows that LFLIU can effectively enhance the permeability of M. smegmatis cell wall, thereby enhancing the bactericidal effect of the antibiotic levofloxacin on $\mathrm{M}$. smegmatis[22]. Our previous work also demonstrates that the synergistic antifungal efficacy of LFLIU combined with amphotericin B-loaded PLGA nanoparticles on C. albicans infection was successfully supported by in vitro and in vivo assays[23, 24].

To design more effective strategies against Mtb, we developed PLGA nanoparticles encapsulating a conventional anti-TB drug(levofloxacin), and observed the combined effect of LFLIU and drug-loaded nanoparticles against intracellular M. smegmatis bacteria based on an in vitro macrophage infection model. It is well known that Mtb grows very slowly, has a strong contagious and pathogenicity, and laboratories must meet the requirements of the National Biosafety Level 3 (BSL-3) in order to carry out a large number of viable Mtb operations. There are more than 2,000 homologous genes between Mtb and M. smegmatis. M. smegmatis is a fast-growing and non-pathogenic species which are often used as a substitute of Mtb in Mtb experimental studies [25]. Therefore, M. smegmatis was selected as the experimental strain in this experiment. In this study, the bactericidal effect and mechanism of LFLIU combined with levofloxacin-loaded PLGA nanoparticles on M. smegmatis in macrophages were investigated. The results provide a new non-invasive, safe, and effective method for the treatment of tuberculosis.

\section{Materials And Methods}




\subsection{Chemicals}

Polyvinyl alcohol (PVA), 3-(4,5-dimethyl-2-thiazolyl)-2,5-diphenyl-2-H-tetrazolium bromide (MTT), levofloxacin (LEV), isopropanol, dichloromethane (DCM), dimethyl sulfoxide (DMSO), sodium dodecyl sulfate (SDS), Tween-80 were purchased from Sigma-Aldrich (St. Louis, MO, USA). Poly (lactide-coglycolic) acid (PLGA) polymer material with a molecular weight of $21 \mathrm{kDa}$ (ratio of lactide to glycolic acid molar ratio of 50:50) was purchased from Rui Jia Biological (Xi'an, China). Dulbecco's Modified Eagle's Medium (DMEM), fetal bovine serum (FBS), trypsin, phosphate buffered saline (PBS) were obtained from Thermo Fisher Scientific (Waltham, MA, USA). Penicillin-streptomycin solution, reactive oxygen species (ROS) assay kit, 1,1'-dioctadecyl-3,3,3',3'-tetramethylindocarbocyanine perchlorate (Dil), 4,6-Diamidino-2phenylindole (DAPI) were obtained from Beyotime Biotechnology Co., Ltd. (Shanghai, China). Middlebrook's 7H9 broth medium, Luria-Bertani (LB) broth, Oleic acid-albumin-dextrose-catalase (OADC) were purchased from BD biosciences (New York, USA).

\subsection{Cell and bacterial culture experiments}

Mouse peritoneal macrophages RAW264.7 were purchased from Shanghai Institute of Cells, Chinese Academy of Sciences and cultured in a humidified incubator in the setting of the partial pressure of $5 \%$ $\mathrm{CO}_{2}$ at $37^{\circ} \mathrm{C}$ in DMEM, which was supplemented with $1 \%$ penicillin/streptomycin and $10 \% \mathrm{FBS}$. RAW264.7 cells were generally seeded in cell culture flask (Corning, USA) for $12 \mathrm{~h}$ to adhere. Then, they were harvested by $0.25 \%$ trypsin-EDTA solution for 2 min for the following experiments.

The bacterial strain used in this study was M. smegmatis mc2155 (purchased from the National Institute for the Control of Pharmaceutical and Biological Products, Beijing, China), an acid-fast bacterial species, which is considered to be a model organism for researching Mtb in the laboratory[25]. The bacteria were grown in Middlebrook's $7 \mathrm{H} 9$ broth medium supplemented with $10 \%$ OADC and $0.05 \%$ Tween- 80 at $37^{\circ} \mathrm{C}$ for $24 \mathrm{~h}$ with agitation $(180 \mathrm{rpm})$. The bacteria were allowed to reach the exponential phase with an optical density $\left(\mathrm{OD}_{600}\right)$ of 0.6-0.8 and were harvested and re-suspended with PBS to a concentration of $10^{6} \mathrm{CFU} / \mathrm{mL}$ for following experiments. The minimally inhibitory concentration (MIC) of LEV against M. smegmatis was determined by micro-broth dilution method.

\subsection{Preparation and characterization of the nanoparticles}

LEV loading PLGA nanoparticles (LEV-NPs) were prepared by the double emulsification method using sonication, as previously described[26]. Briefly, PLGA was dissolved completely in DCM. A preweighed amount of LEV was dissolved in acetic acid that was miscible with water $(20: 80 \%, \mathrm{v} / \mathrm{v})$. The dissolved PLGA polymer material and the drug were mixed for the first ultrasonic sonicated using an ultrasonic processor (XL2020, USA) in an ice bath at $100 \mathrm{~W}$ ultrasonic power for $2 \mathrm{~min}$. Next, the 1\% PVA cooled was added to the polymeric mixture for the second ultrasonic sonicated in an ice bath at $100 \mathrm{~W}$ ultrasonic power for $5 \mathrm{~min}$. After that, $2 \%$ isopropanol was added to the suspension, followed by magnetic stirring at room temperature for $4 \mathrm{~h}$ to evaporate the DCM. The LEV-NPs were washed and collected by centrifugation at $8000 \mathrm{~g}$ for $10 \mathrm{~min}$. After that, the LEV-NPs were lyophilized in a freeze dryer (Christ 
ALPHA 2-4 LSC plus,Osterode匹Germany) for the following study. Plain PLGA nanoparticles (NPs) and Dil loading PLGA nanoparticles (Dil-NPs) were prepared following a similar method, except that the drug was exchanged for an equal amount of deionized water or Dil (Final concentration $10 \mu \mathrm{M}$ ).

The average diameter, zeta potential, and polydispersity index (PDI) were determined by dynamic light scattering (DLS) using Malvern laser particle size analyzer (Zeta SIZER 3000HS, USA). The morphological characterization of nanoparticles was observed by transmission electron microscopy (TEM, Hitachi HighTechnologies, Tokyo, Japan) and scanning electron microscopy (SEM, Hitachi High-Technologies, Tokyo, Japan).

\subsubsection{Determination of LEV-NPs loading content and encapsulation efficiency}

$2 \mathrm{mg}$ of freeze-dried nanoparticles was dissolved in $1 \mathrm{~mL}$ of DCM, and the drug concentration was determined by an UV-Vis spectrophotometer (UV-2600 SHIMADZU, Japan) at $330 \mathrm{~nm}$. The drug loading content (LC) and encapsulation efficiency (EE) were calculated using the following equation:

LC $(\%)=[$ weight of the drug in nanoparticles / weight of the nanoparticles ] $\times 100 \%$,and

$\mathrm{EE}(\%)=[$ weight of the drug in nanoparticles / weight of the feeding drug $] \times 100 \%$

\subsubsection{In vitro ultrasound-triggered LEV release from LEV- NPs}

The kinetic release of LEV from LEV-NPs in vitro with sonication was investigated. A sample of LEV-NPs lyophilized powder was diluted in PBS with sonication (fixed frequency of $42 \mathrm{kHz}$ ) at an intensity of $0.13 \mathrm{~W} / \mathrm{cm}^{2}$ for $10 \mathrm{~min}$. After sonication, the samples were then transferred into dialysis bags(MWCO: $7000 \mathrm{Da}$,Biosharp, Hefei, China), which was incubated in $50 \mathrm{~mL}$ PBS and shaken at $100 \mathrm{rpm}$. Dialysate samples $(1 \mathrm{~mL})$ were collected, and the percentage of LEV released from nanoparticles was evaluated by an UV-vis spectrophotometry (UV-2600 SHIMADZU, Japan) at each predetermined time point. Dialysate samples from LEV-NPs that did not undergo sonication were used as controls. The cumulative drug release was calculated using the following equation:

Cumulative release $(\%)=$ [weight of LEV released from LEV-NPs / initial weight of the drug in LEV-NPs] $\times$ $100 \%$.

\subsection{Ultrasonic irradiation method}

The LFLIU system device used in this experiment was developed by the Chongqing Medical University Institute of Biomedical Engineering, with a transducer diameter of $45 \mathrm{~mm}$, a fixed frequency of $42 \mathrm{kHz}$, adjustable ultrasonic intensity output of $0.13 \mathrm{~W} / \mathrm{cm}^{2}$ to $0.33 \mathrm{~W} / \mathrm{cm}^{2}$. The acoustic field was measured with a hydrophone (Onda Corp, Sunnyvale, CA, USA). The medical ultrasonic couplant was uniformly coated on the top of the transducer. The $35 \mathrm{~mm}$ cell culture dish was placed directly above the transducer and gently squeezed to expel the air, followed by ultrasound irradiation (as shown in Figure.1) with the 
working mode of a continuous-wave. In this study, the dose of ultrasound at the intensity of $0.13 \mathrm{~W} / \mathrm{cm}^{2}$ with irradiation for 10 minutes has been chosen due to little effect on the macrophage activity(based on the preliminary data). Before the experiment, all samples were equilibrated at room temperature $\left(25^{\circ} \mathrm{C}\right)$ by air conditioners. The temperature of the cell suspension was monitored through a needle thermosensors with digital-display (batch 119, No. 02810232; Yuyao Temperature Instrument Factory Co, Ltd, China).

\subsection{Cytotoxicity assay}

The cytotoxicity of LEV and LEV-NPs against RAW264.7 cells were investigated by MTT assay. Briefly, RAW264.7 cells $\left(1 \times 10^{5}\right.$ cells $\left./ \mathrm{mL}\right)$ were grown in Petri dish for $24 \mathrm{~h}$ to allow cells to adhesion, then treated with the LEV and LEV-NPs at containing final drug concentration of $0 \mu \mathrm{g} / \mathrm{mL}, 2 \mu \mathrm{g} / \mathrm{mL}, 4 \mu \mathrm{g} / \mathrm{mL}$, $8 \mu \mathrm{g} / \mathrm{mL}, 16 \mu \mathrm{g} / \mathrm{mL}, 32 \mu \mathrm{g} / \mathrm{mL}, 64 \mu \mathrm{g} / \mathrm{mL}, 128 \mu \mathrm{g} / \mathrm{mL}, 256 \mu \mathrm{g} / \mathrm{mL}$ )for 4 hours, respectively. After that, the cells were continuously irradiated for $10 \mathrm{~min}$ with $0.13 \mathrm{~W} / \mathrm{cm}^{2}$ ultrasound (The ultrasound dose used is based on the preliminary data), and then cells were cultured for another $24 \mathrm{~h}$. Then the MTT experiment was carried out. The control group consisted of the same procedure but no ultrasound treatment. The relative cell viability was calculated as follows: cell viability $(\%)=\mathrm{OD}_{570}$ (treatment) $/ \mathrm{OD}_{570}$ (control) $\times$ $100 \%$. The values are presented as averages of three independent experiments.

\subsection{Phagocytosis of macrophages on nanoparticles}

In this study, Dil-NPs was used as a model to study the phagocytic effect of macrophages on LEV-NPs under ultrasound. The nucleus of RAW264.7 cells were stained with DAPI (blue fluorescence, $10 \mu \mathrm{g} / \mathrm{mL}$ ). Dil-NPs (red fluorescence, $4 \mu \mathrm{g} / \mathrm{mL}$ ) were added to the culture dish of RAW264.7 cells, then irradiated with $0.13 \mathrm{~W} / \mathrm{cm}^{2}$ ultrasound for $10 \mathrm{~min}$, and that without ultrasonic irradiation were taken as control. After incubation for another 3 hours, the plates were washed three times to remove the extracellular Dil-NPs. RAW264.7 cells were observed by a laser confocal microscopy (CLSM, A1 + R, Nikon, Tokyo, Japan) at excitation/emission wavelengths of $364 / 454 \mathrm{~nm}$ for DAPI and excitation/emission wavelengths of $549 / 565 \mathrm{~nm}$ for Dil. In addition, the relative fluorescence intensity of intracellular Dil-NPs was quantified using flow cytometry(CytoFLEX, Beckman Coulter, Inc. CA, USA).

\subsection{Intracellular killing of M. smegmatis and TEM observation}

The killing efficiency of ultrasound combined with nanoparticles treatment on M. smegmatis in macrophages was investigated. $10^{5}$ RAW264.7 cells per well were seeded in Petri dish and allowed to grow for $24 \mathrm{~h}$. After washed thrice with antibiotics-free medium, added fresh cultured M. smegmatis (ratio of bacteria/cells:10:1) into Petri dish to infect the cells for $2 \mathrm{~h}$, then washed thrice to remove the extracellular bacteria. RAW264.7 cells infected with M. smegmatis alone without ultrasound treatments were used as controls. Subsequently, the infected macrophages were incubated in DMEM for their exposure to the LEV-NPs (where LEV was at a final concentration of $4 \mu \mathrm{g} / \mathrm{mL}$ ) and the free LEV (at a final 
concentration of $4 \mu \mathrm{g} / \mathrm{mL}$ ). After that, the macrophages were irradiated with ultrasonic intensity of $0.13 \mathrm{~W} / \mathrm{cm}^{2}$ for $10 \mathrm{~min}$, cultured for another $3 \mathrm{~h}$ at $37^{\circ} \mathrm{C}$ in an atmosphere of $5 \% \mathrm{CO}_{2}$, the extracellular free LEV and LEV-NPs were washed with PBS. After treatment for $12 \mathrm{~h}$, cells were washed and collected. One part of cell samples after treatment were made into ultrathin sections for observation of the internal structure of macrophages by transmission electron microscopy (TEM, JEM-1400PLUS, Hitachi HighTechnologies, Tokyo, Japan); other part of cell samples were lysed using distilled water containing $0.25 \%$ SDS for observation of the intracellular bacteria and evaluation of bacterial activity. The survival of intracellular bacteria was estimated by plating serially diluted cultures on $7 \mathrm{H} 10$ plates, and the colonyforming units (CFU) were enumerated after $48 \mathrm{~h}$. All samples were plated in triplicate, and values were averaged from three independent trials. Similarly, the intracellular bacteria were also made into ultrathin sections for TEM observation.

\subsection{Quantitation of intracellular reactive oxygen species}

Intracellular ROS was analyzed using flow cytometer(CytoFLEX, Beckman Coulter, Inc. CA, USA)with a ROS reagent kit with fluorescent probe DCFH-DA. DCFH-DA itself has no fluorescence, and after entering the cell, it was hydrolyzed by the esterase in the cell to form dichlorofluorescin (DCFH). Intracellular ROS could oxidize non-fluorescent DCFH to produce fluorescent dichlorofluorecein (DCF), which was impermeable to the cell membrane. Therefore, the level of ROS in the cells can be known by detecting the fluorescence of the DCF. Briefly, infection RAW264.7 cells were incubated with DCFH-DA (final concentration $10 \mu \mathrm{M})$ for $30 \mathrm{~min}$. The nucleus was blue stained with DAPI $(10 \mu \mathrm{g} / \mathrm{mL})$ for $10 \mathrm{~min}$. After that, the cells were washed and incubated in DMEM for exposure to the LEV-NPs ( drug concentration of $4 \mu \mathrm{g} / \mathrm{mL})$ and the free LEV $(4 \mu \mathrm{g} / \mathrm{mL})$ and treated with ultrasound at the intensity of $0.13 \mathrm{~W} / \mathrm{cm}^{2}$ for $10 \mathrm{~min}$, the treated cells were collected, resuspended in serum-free medium, and measured using the flow cytometer with the excitation setting at $488 \mathrm{~nm}$. The obtained data was analyzed using Cell Lab Quanta SC MPL Analysis software (CytoFLEX, Beckman Coulter, CA, USA). In addition, the level of intracellular ROS production was observed using a laser confocal microscope (CLSM, A1 + R, Nikon, Tokyo, Japan) at excitation/emission wavelengths of $364 / 454 \mathrm{~nm}$ for DAPI and excitation/emission wavelengths of $488 / 525 \mathrm{~nm}$ for DCF. Without ultrasound irradiation, the others were treated with the same method as control. Experiments were repeated three times independently.

\subsection{Apoptosis and necrosis of RAW264.7 cells}

Annexin V-FITC/PI double staining kit was used to detect apoptosis and necrosis of RAW264.7 cells under different treatments: control (no drug, no ultrasound), free LEV(only LEV), ultrasound (US), ultrasound combined with free LEV (US + LEV), LEV-NPs, ultrasound combined with LEV-NPs (US + LEVNPs). The drug concentration in LEV group and LEV-NPs group were $4 \mu \mathrm{g} / \mathrm{mL}$ based on MIC. The ultrasonic dose used was $0.13 \mathrm{~W} / \mathrm{cm}^{2}$ for $10 \mathrm{~min}$ (based on preliminary data). After the treatment was completed, the cells were incubated for another 24 hours. The treated cells were collected and resuspended in $1 \mathrm{~mL}$ PBS while adding $5 \mu \mathrm{L}$ of Annexin V-FITC and $10 \mu \mathrm{L}$ of PI, the dye was mixed and 
incubated at room temperature for $15 \mathrm{~min}$ in a dark environment. The resulting samples were detected by flow cytometry (CytoFLEX, Beckman Coulter, Inc. CA, USA). The obtained data was analyzed using Cell Lab Quanta SC MPL Analysis software (CytoFLEX, Beckman Coulter, CA, USA). Experiments were repeated three times independently.

\subsection{Statistics analysis}

Results were analyzed using one-way ANOVAs in SPSS 17 statistical software (IBM, Chicago, USA). The data were expressed as mean \pm standard deviation. $\mathrm{P}<0.05$ was considered statistically significant.

\section{Results}

\subsection{Characterization of nanoparticles}

The physical properties of blank NPs and LEV-NPs were presented in Figure. 2. SEM imaging indicated that the blank NPs and LEV-NPs exhibited a smooth and uniform spherical morphology (Fig. 2A and 2C). The center of the blank NPs showed white under TEM, while the LEV-NPs showed an enhanced dark black area (Fig. 2B and 2D). This proved that the LEV was loaded into the PLGA shell.

Table 1 shows the results of the LC and the EE of LEV-NPs ( $8.36 \pm 0.74 \%$ and $84.74 \pm 1.28 \%$, respectively). Besides, size distribution, zeta potential, PDI of NPs and LEV-NPs were compared in this study. The average diameter of the LEV-NPs was $229.8 \pm 12.1 \mathrm{~nm}$, with a polydispersity index (PDI) of $0.038 \pm 0.007$, and the microcapsule surface was negatively charged (Zeta potential is an important factor for nanoparticle stability. A reasonable zeta potential can prevent the NPs from aggregation[27]). The particle size of LEV-NPs was greater than that of NPs, and the difference was statistically significant $(P<0.05)$. In short, the above results prove that we successfully prepared LEV-NPs with uniform size, and stable properties for the delivery of LEV.

Table 1

The physical characteristics of the developed nanoparticles

\begin{tabular}{|llllll|}
\hline Formulations & Particle size $(\mathrm{nm})$ & Zeta potential (mV) & PDI & LC (\%) & EE (\%) \\
\hline Blank NPs & $173.1 \pm 13.2$ & $-20.8 \pm 4.25$ & 0.134 & - & - \\
\hline LEV-NPs & $229.8 \pm 12.1 *$ & $-28.8 \pm 3.78$ & 0.038 & $8.36 \pm 0.74$ & $84.74 \pm 1.28$ \\
\hline $\begin{array}{l}\text { Notes: NPs, PLGA nanoparticles; LEV-NPs, Levofloxacin-loaded nanoparticles; PDI, polydispersity } \\
\text { index; LC, loading content; EE, encapsulation efficiency. Standard deviation for } \mathrm{n}=3 \text {. *P }<0.05 \\
\text { compared with NPs. }\end{array}$ & & & \\
\hline
\end{tabular}

3.2 In vitro investigations of ultrasound-triggered drug release 
The LEV release from LEV-NPs was assessed by performing in vitro ultrasound-triggered experiments. Figure 3 displays the drug release curves of LEV-NPs with and without ultrasound. Drug release occurs in two phases: an initial "burst" release phase and subsequent slower release. Results showed that the release rate of LEV with sonication was faster than that without sonication. For the LEV-NPs with ultrasound,approximately $74 \%$ of the LEV was released after $72 \mathrm{~h}$. However, for the LEV-NPs without ultrasound, approximately $39 \%$ of the encapsulated LEV was released after $72 \mathrm{~h}$. This result implies that the LEV-NPs can be controllably triggered with ultrasound. PLGA shells may be destroyed by ultrasound to promote the diffusion of LEV, resulting in increased drug release rates.

\subsection{Cytotoxicity of LEV-NPs on macrophage cells}

In vitro cytotoxicity experiment was carried out in RAW264.7 cells using the MTT assay. Figure 4 showed cytotoxicity of free LEV and LEV-NPs with or without ultrasonic treatment on RAW264.7 cells. The cell viability decreased to $68.52 \pm 5.46 \%$ when the cells were treated by free LEV alone at the concentration of $64 \mu \mathrm{g} / \mathrm{mL}$. Free LEV combined with ultrasound treatment, cell viability began to decline when the concentration of free levofloxacin was $8 \mu \mathrm{g} / \mathrm{mL}$, which was $74.18 \pm 8.58 \%$. For both cases, with the increase of concentration of free drug, the decline of cell viability is more serious. LEV-NPs alone at drug concentrations from $0 \mu \mathrm{g} / \mathrm{mL}$ to $256 \mu \mathrm{g} / \mathrm{mL}$ was not cytotoxic to RAW264.7 cells. When LEV-NPs combined with ultrasound treatment, cell viability began to decrease when the drug concentration in nano-preparation was $128 \mu \mathrm{g} / \mathrm{mL}$, which was $69.84 \pm 4.26 \%$. As it was visible, at the same drug concentration and with or without ultrasound, the toxicity of LEV-NPs to RAW264.7 cells was lower than that of free drugs.

\subsection{LFLIU promoted phagocytosis of drug-loaded nanoparticles}

Dil-NPs(red fluorescence)were used as a model to evaluate the macrophage phagocytosis to nanoparticles. The red fluorescence intensity of intracellular Dil-NPs was observed following different treatments. Figure 5 shows the phagocytosis of Dil-NPs after RAW264.7 cells were treated with Dil-NPs or combined with ultrasound. A laser confocal microscope can be seen that the Dil-NPs (red) are tightly surrounded around the nucleus (blue). The flow cytometry analysis showed that the relative fluorescence intensity of the Dil-NPs group was $33.18 \pm 4.16 \%$, while the phagocytosis rate of the ultrasound combined with Dil-NPs group was $54.86 \pm 7.45 \%$. Obviously, the phagocytosis rate of the ultrasound combined with the Dil-NPs group was higher than that of the Dil-NPs group $(P<0.05)$.

\subsection{Intracellular bacterial vitality post-treatment}

Fighting intracellular pathogens is a major challenge due to the fact that cell membrane/wall is a critical barrier for a drug used entering difficultly the cells to kill the bacteria. Since mycobacterium is an intracellular pathogen, and resistant to numerous drugs due to lipid-rich cell wall [28]. We determined the intracellular burden of M. smegmatis in macrophages after treated with free LEV, LEV-NPs, ultrasound 
(US), ultrasound combined with LEV(US + LEV), ultrasound combined with LEV-NPs (US + LEV-NPs). As shown in Fig. 6, at 12 hours after the treatments, US + LEV-NPS, US + LEV, LEV-NPs, and US killed 82.2\%, $48.1 \%, 23.7 \%$ and $30.0 \%$ of M. smegmatis, respectively, whereas free LEV alone killed $7.8 \%$ of bacteria under similar conditions compared to the control. Obviously, ultrasound combined with LEV-NPs resulted in an approximately 2-fold decrease of the intracellular bacterial burden as compared to ultrasound combined with free LEV.

\subsection{The cell structure following treatment}

The TEM observation of RAW 264.7 cells and intracellular M. smegmatis following different treatments were shown in Fig. 7. The cells in control were intact with abundant cytoplasm, mitochondria which were apparent, cell membranes and the nuclear envelope that were intact, and nuclear materials which were uniform. The morphology and structure of cells in the free LEV group was similar to that in control. In the LEV-NPs group, RAW 264.7 cells were injured to some extent, and a small number of cells displayed limited microvilli, cytoplasm became less dense, LEV-NPs (red arrow) can be seen around the nucleus. In the US group, the macrophage structure was relatively intact, but autophagosomes (white arrows) can be observed. In the LEV + US group, the cell morphology was significantly changed, cells were severely damaged, a portion of cell microvilli had vanished, the volume of the cell nucleus had decreased,and chromatin was gathered densely. In addition, lipid droplets (yellow arrow) were clearly visible. In the LEVNPs + US group, the volume of the cell nucleus had more decreased compared with other treatments, and chromatin was condensed in nuclear, thus presenting characteristics of apoptosis. It is worth noting that drug-loaded nanoparticles (blue arrows) in M. smegmatis were observed.

Figure 8 showed the observation of bacterial damage when M. smegmatis was extracted from the lysis of macrophages by different treatments. Bacterial cell wall integrity in the control group and the LEV group. In the LEV-NPs group, part of the cytoplasm of M. smegmatis was not dense, and LEV-NPs (black arrows) within the bacteria were observed. There was no significant change in bacterial morphology in the US group. However, in the LEV + US group, bacterial swelling, bacterial lipid droplets, and glycogen (yellow arrow) were significantly increased compared with the control group. In LEV-NPs + US group, the bacterial cell wall is broken (blue arrow), and cell structure is incomplete, indicating that the cells were severely damaged. What 's more, LEV-NPs in M. smegmatis were also observed (red arrow).

\subsection{Intracellular ROS level following treatment}

The intracellular generation of ROS was observed by laser scanning confocal microscopy and flow cytometry with DCFH-DA (Fig. 9). Figure 9A qualitatively observed that the ROS level (green fluorescence) of the LEV group, the LEV-NPs group, the US group, the LEV + US group, and the LEV-NPs + US group were higher than that of the control group. The results of the quantitative analysis of the ROS level by flow cytometry in Fig. 9B were consistent with those in Fig. 9A, and the fluorescence intensity of the treatment group was higher than that of the control group $(P<0.05)$. The results showed that the green fluorescence 
intensity of the LEV-NPs + US group was the highest, reaching $1844.3 \pm 46.7$, which was about twice that of the control group $(993.9 \pm 47.5)$.

\subsection{Apoptotic and necrosis of macrophage following treatment}

The apoptotic rate and necrosis rate of RAW264.7 cells are shown in Fig. 10 and Table 2. Both apoptosis and necrosis were observed in macrophages, and the apoptotic ratio was higher than the necrotic ratio under appropriate conditions. Compared with the control group, the apoptosis rates of the LEV group, the LEV-NPs group, the US group, the LEV + US group, and the LEV-NPs + US group were increased. The maximum apoptosis ratio $(21.25 \pm 1.15) \%$ was observed in the LEV-NPs + US group. Compared with the control group, the necrosis rate of the LEV-NPs group, the US group, the LEV + US group, and the LEV-NPS + US group increased. It is worth noting that there was a nearly equal cell necrotic ratio in the US group, LEV + US group, and LEV-NPS + US group.

Table 2

Necrosis rate and apoptotic rate of RAW264.7 cells under different treatments.

\begin{tabular}{|c|c|c|c|}
\hline Groups & $\mathbf{N}$ & Apoptosis rate & Necrosis rate \\
\hline control & 6 & $3.30 \pm 1.01$ & $2.21 \pm 0.21$ \\
\hline LEV & 6 & $6.60 \pm 1.78^{*}$ & $2.28 \pm 0.35$ \\
\hline LEV-NPS & 6 & $10.42 \pm 0.84^{* \#}$ & $4.20 \pm 1.04^{* \#}$ \\
\hline US & 6 & $8.76 \pm 0.78^{*}$ & $9.54 \pm 0.88^{\star \#}$ \\
\hline LEV + US & 6 & $15.54 \pm 1.30^{* \#}$ & $7.82 \pm 044^{\star \#}$ \\
\hline LEV-NPS + US & 6 & $21.25 \pm 1.15^{\star \#}$ & $8.21 \pm 0.66^{* \#}$ \\
\hline \multicolumn{4}{|c|}{$\begin{array}{l}\text { Notes: Compared to the control, }{ }^{*} \mathrm{P}<0.05 \text {; compared to the LEV, }{ }^{\#} \mathrm{P}<0.05 \text {. Data represent the mean } \pm \\
\text { SD. }\end{array}$} \\
\hline \multicolumn{4}{|l|}{ Abbreviations: } \\
\hline \multicolumn{4}{|c|}{$\begin{array}{l}\text { LEV, levofloxacin; LEV-NPs, levofloxacin-loaded PLGA nanoparticles; US, ultrasonic; SD, standard } \\
\text { deviation. }\end{array}$} \\
\hline
\end{tabular}

\section{Discussion}

Nanomedicine has dramatically changed the concept of traditional medicines for treating diseases, and many nanomedicine delivery systems have shown great promise[29-31]. Especially, the application of ultrasound combined with drug-loaded nanoparticles is a hotspot of many scholars. In recent years, ultrasound combined with drug-loaded nanoparticles has been widely reported in the treatment of tumors, killing bacteria, inhibiting biofilm growth[32-34]. 
The biodegradable polymeric nanoparticle-encapsulated formulations as an efficient and promising tool for delivering therapeutic molecules to the infected tissue, and this technique is particularly useful to improve the anti-infection effect[35-37]. In this study, we successfully prepared levofloxacin-loaded nanoparticles by PLGA copolymer using a double-emulsification method. The average particle size was only $229.8 \pm 12.1 \mathrm{~nm}$, and the size was uniform (see Fig. 2). We compared the cytotoxicity of LEV-NPs and free LEV on macrophages RAW264.7 with or without ultrasound. The results showed that when the concentration of free LEV reached $8 \mu \mathrm{g} / \mathrm{mL}$, the cell viability decreased to $68.52 \pm 5.46 \%$. With the increase of drug concentration, the damage of cell activity is further aggravated. Nevertheless the drug concentration in the LEV-NPs reached $64 \mu \mathrm{g} / \mathrm{mL}$ even with ultrasound, showed no cytotoxicity, indicating that the nanoparticles reduced the toxic effect of the drug on the cells (see Fig. 4).

Ultrasound triggered the release of LEV from polymeric nanoparticles was studied in this study. In this experiment, the natural release rate of LEV from drug-loaded nanoparticles in 72 hours is only about $20 \%$, which proves that it is a long-term slow-release process. However, the drug release rate from nanoparticles increased nearly twice at the same time after irradiation with a certain dose of lowfrequency ultrasound. The results indicate that low-frequency ultrasound irradiation could promote the release of the contents from the drug-loaded nanoparticles (Fig. 3). This result is consistent with earlier studies that demonstrated low-frequency acoustic activity could stimulate the release of therapeutic substances from nanoparticle formulations increasing the local concentration of drugs and shorten the treatment period[38].

Previous literature reported that low-frequency ultrasound $(20-100 \mathrm{kHz})$ produced a series of "acoustic biological effects" dominated by acoustic cavitation, instead of thermal effects. Cavitation events occurring during ultrasound irradiation is the key mechanism of sonoporation[39-41]. Low frequency acoustic cavitation-induced sonoporation offers a noninvasive method of drug delivery. Ultrasonic sonoporation is the temporary opening in the cell membrane/wall, which increases the permeability of the cell plasma membranes and allows the exchange of substances between inside and outside the cell[42, 43]. In addition, nanoparticles may act as cavitation nuclei enhances the sonoporation effect, which is helpful to increase cell membrane permeability and drug intake further[44, 45].

The results in this study confirmed that the joint use of ultrasound and drug-loaded nanoparticles had synergetic bactericidal efficacy on $\mathrm{M}$. smegmatis in macrophage, compared to the other treatments. The action of ultrasound is beneficial to realize the delivery effectively of the LEV-NPs to the M. smegmatis in macrophages and increase the drug concentration in the cell to kill intracellular M. smegmatis. This experiment on ultrasound promoting macrophage to uptake nanoparticles (see Fig. 5) also confirmed this argument indirectly. In addition, we observed a significant increase in lipid droplets and glycogen in $\mathrm{M}$. smegmatis, and an incomplete cell wall rupture structure following LEV-NPs + US (Fig. 8). All these indicate that ultrasound combined with drug-loaded nanoparticles caused the most serious damage of M. smegmatis. It can be explained that the rupture of nanoparticles enhances the acoustic cavitation effect through some complex dynamics during ultrasound irradiation, which further increases the cell 
wall permeability and more effectively promotes the drug to enter the cells, thus improving the bactericidal effect.

We observed under TEM that in the LEV-NPS + US group, the volume of nucleus decreased and chromatin condensed, showing the characteristics of apoptosis, which was consistent with the results of direct analysis by flow cytometry, where the highest apoptotic rate was observed (21.25 $\pm 1.15 \%)$ (see Fig. 10 and Table 2) in the LEV-NPs + US group. Ultrasound promotes the apoptosis of macrophages, and the apoptosis of macrophages itself is conducive to the death of intracellular bacteria.

Apoptosis is an innate macrophage defense mechanism. Apoptosis of infected macrophages is associated with diminished pathogen viability. The concept supported by the finding that apoptosis reduces the viability of intracellular bacillus Calmette-Guerin and Mtb[46, 47]. Necrosis can also be observed in infected macrophages of some treatment groups (Fig. 10 and Table 2). Necrosis is a mechanism used by bacteria to exit the macrophage, evade host defenses, and spread[48, 49]. This seems to be a conflict about the fate of intracellular M. smegmatis if apoptosis and Necrosis were induced in infected macrophages underwent different treatments. In this study, the necrosis ratio in some groups(e.g. LEV-NPs + US, LEV + US) increases slightly, and the apoptosis ratio increase more significantly. These results indicate that ultrasound combined with drug-loaded nanoparticles can induce apoptosis more obviously and help to decrease intracellular bacterial viability.

Beyond the above mechanism, another possible mechanism of enhanced antibacterial action may be related to increased the intracellular activity of reactive oxygen species (ROS). The stimulated generation of ROS after ultrasound irradiation is toxic to microorganism and has significant antimicrobial activity against planktonic and biofilm forms[24,50]. The production of ROS was qualitatively and quantitatively analyzed by confocal laser microscopy and flow cytometry (see Fig. 9) in this study. The intracellular ROS levels in the group of the combination of ultrasound and LEV-NPs are highest among all groups.

All over, our study shows that ultrasound-assisted LEV-NPs can effectively inactivate intracellular M. smegmatis. The death of $\mathrm{M}$. smegmatis in macrophages is related to cavitation events occurring, apoptosis of macrophages, and the level of ROS produced in macrophages during ultrasound irradiation. The combination of ultrasound with drug-loaded nanoparticles can be considered as a prospective strategy for tuberculosis therapy to achieve drug delivery efficiently for effectively killing MTB and expected to shorten the course of chemotherapy for tuberculosis significantly.

\section{Declarations}

\section{Acknowledgements}

Not applicable

\section{Authors' contributions}


Dairong Li and Yonghong Du conceived and designed research; Shuang Xie and Gangjing Li performed experiments; Yuru Hou and Min Yang analyzed data; Shuang Xie prepared figures; Shuang Xie and Gangjing Li drafted manuscript; Fahui Li and Jianhu Li search literature; Shuang Xie and Dairong Li edited and revised manuscript; Dairong Li, Yonghong Du and Shuang Xie approved final version of manuscript.

\section{Funding source}

This work was supported by the Chongqing Research Program of Basic Science and Frontier Technology (No. csct2016jcyjA0098), and the Program of Chongqing Special Social Livelihood of the People of Science and Technology Innovation (No cstc2019jscx-msxmX0255). The funders above did not have any involvement in the study design, data collection, data analysis, interpretation, and manuscript writing.

\section{Availability of data and materials}

The data, analytical methods, and study materials for the purposes of reproducing the results or replicating procedures can be made available on request to the corresponding author who manages the information

\section{Consent for publication}

Not applicable.

\section{Declaration of competing interest}

The authors declare no competing interests with relevance to this study.

\section{Author details}

${ }^{1}$ State Key Laboratory of Ultrasound in Medicine and Engineering, College of Biomedical Engineering, Chongqing Medical University, Chongqing, 400016, China. Chongqing Key Laboratory of Biomedical Engineering, Chongqing Medical University, Chongqing, 400016, China. ${ }^{2}$ Department of Respiratory and Critical Care Medicine, the First Affiliated Hospital of Chongqing Medical University, Chongqing 400016, China.

\section{References}

[1] Sushruta H, Prasad SV, Santosh G, et al. Antimycobacterial susceptibility evaluation of rifampicin and isoniazid benz-hydrazone in biodegradable polymeric nanoparticles against Mycobacterium tuberculosis H37Rv strain. International Journal of Nanomedicine 2018;13:4303-18.

[2] Hockhausen K, Odegaard K, Boersma B, et al. A Review of Extrapulmonary Tuberculosis 2018;71:116119. 
[3] Sotgiu G, Sulis G, Matteelli A. Tuberculosis-a World Health Organization Perspective. Microbiology Spectrum 2017;5.

[4] Global tuberculosis report 2019. Geneva: World Health Organization; 2019.

[5] Toit LCD, Pillay V, Danckwerts MP. Tuberculosis chemotherapy: current drug delivery approaches. Respiratory Research 2006;7:118.

[6] Ariel PM, Gowda DK, Frieden TR. Controlling multidrug-resistant tuberculosis and access to expensive drugs: a rational framework. Bull World Health Organ 2002;80:489-95.

[7] Ahuja SD, David A, Monika A, Rita B, et al. Multidrug resistant pulmonary tuberculosis treatment regimens and patient outcomes: an individual patient data meta-analysis of 9,153 patients. PLoS Medicine, 2012; 9: e1001300.

[8] Kalhapure RS, Nadia S, Chunderika M, et al. Nanoengineered drug delivery systems for enhancing antibiotic therapy. Journal of Pharmaceutical Sciences 2015;104:872-905.

[9] Huh AJ, Kwon YJ. "Nanoantibiotics": a new paradigm for treating infectious diseases using nanomaterials in the antibiotics resistant era. Journal of Controlled Release 2011;156:128-45.

[10] Shegokar R, Al SL, Mitri K. Present status of nanoparticle research for treatment of tuberculosis. Journal of Pharmacy \& Pharmaceutical Sciences 2011;14:100-16.

[11] Abed N, Couvreur P. Nanocarriers for antibiotics: a promising solution to treat intracellular bacterial infections. Int J Antimicrob Agents 2014;43:485-96.

[12] Cheow WS, Hadinoto K. Antibiotic Polymeric Nanoparticles for Biofilm-Associated Infection Therapy. Methods in Molecular Biology 2014;1147:227-38.

[13] Gao W, Chen Y, Zhang Y, et al. Nanoparticle-based local antimicrobial drug delivery. Adv Drug Deliv Rev 2017:S0169409X17301965.

[14] Chereddy KK, Vandermeulen G, Préat V. PLGA based drug delivery systems: Promising carriers for wound healing activity. Wound Repair \& Regeneration 2016;24:223-36.

[15] Lavon I, Kost J. Ultrasound and transdermal drug delivery. Drug Discovery Today 2004;9:670-6.

[16] Hideo U, Mizue M, Toshinobu S, et al. Acoustic cavitation as an enhancing mechanism of lowfrequency sonophoresis for transdermal drug delivery. Biological \& Pharmaceutical Bulletin 2009;32:916.

[17] Sassaroli E, Hynynen K. Cavitation Threshold of Microbubbles in Gel Tunnels by Focused Ultrasound. Ultrasound in Medicine \& Biology 2007;33:1651-60. 
[18] Miller D, Dou C. Induction of apoptosis in sonoporation and ultrasonic gene transfer. Ultrasound in Medicine \& Biology 2009;35:144-54.

[19] Yu H, Chen S, Cao P. Synergistic bactericidal effects and mechanisms of low intensity ultrasound and antibiotics against bacteria: a review. Ultrasonics - Sonochemistry 2012;19:377-82.

[20] Yu-Ying, Fu, Liang, et al. Synergistic antibacterial effect of ultrasound microbubbles combined with chitosan-modified polymyxin B-loaded liposomes on biofilm-producing Acinetobacter baumannii. International Journal of Nanomedicine 2019; 14:1805-1815.

[21] Wang X, Ip M, Leung AW, et al. Sonodynamic action of curcumin on foodborne bacteria Bacillus cereus and Escherichia coli. Ultrasonics 2015; 62:75-9.

[22] Dong $\mathrm{Y}$, Su $\mathrm{H}$, Jiang $\mathrm{H}$, et al. Experimental study on the influence of low-frequency and low-intensity ultrasound on the permeability of the Mycobacterium smegmatis cytoderm and potentiation with levofloxacin. Ultrasonics Sonochemistry 2017;37:1-8.

[23] Yang M, Xie S, Adhikari VP, et al. The Synergistic Fungicidal Effect of Low-Frequency and LowIntensity Ultrasound with Amphotericin B-loaded Nanoparticles on C. albicans in Vitro. International Journal of Pharmaceutics 2018:S0378517318301807.

[24] Yang M, Du K, Hou Y, et al. Synergistic Antifungal Effect of Amphotericin B-Loaded Poly(Lactic-CoGlycolic Acid) Nanoparticles and Ultrasound against Candida albicans Biofilms. Antimicrobial agents and chemotherapy 2019;63. e02022-18.

[25] Reyrat JM, Kahn D. Mycobacterium smegmatis: an absurd model for tuberculosis? Trends in Microbiology 2001;9:472.

[26] Edagwa BJ, Guo D, Puligujja P, et al. Long-acting antituberculous therapeutic nanoparticles target macrophage endosomes. Faseb Journal 2014;28:5071-82.

[27] Barner A, Myers M. The effects of carboxylic acids on the aqueous dispersion and electrophoretic deposition of ZrO2. Journal of the European Ceramic Society 2013;32:235-44.

[28] Pieters J. Mycobacterium tuberculosis and the Macrophage: Maintaining a Balance. Cell Host \& Microbe 2008;3:399-407.

[29] Snipstad S, Westrøm S, Mørch Y, et al. Erratum to: Contact-mediated intracellular delivery of hydrophobic drugs from polymeric nanoparticles. Cancer Nanotechnol 2015;6:3.

[30] Georgy M, Ursa M, Magaeva AA, et al. Ferri-liposomes as an MRI-visible drug-delivery system for targeting tumours and their microenvironment. Nature Nanotechnology 2011;6:594-602. 
[31] Kisich KO, Gelperina S, Higgins MP, et al. Encapsulation of moxifloxacin within poly(butyl cyanoacrylate) nanoparticles enhances efficacy against intracellular Mycobacterium tuberculosis. Int $\mathrm{J}$ Pharm 2007;345:154-62.

[32] Yu T, Enguo J, Jinsong R, et al. Bifunctionalized mesoporous silica-supported gold nanoparticles: intrinsic oxidase and peroxidase catalytic activities for antibacterial applications. Advanced Materials 2015;27:1097-104.

[33] Dong M, Green AM, Willsey GG, et al. Effects of acoustic streaming from moderate-intensity pulsed ultrasound for enhancing biofilm mitigation effectiveness of drug-loaded liposomes. Journal of the Acoustical Society of America 2015;138:1043-51.

[34] Shen S, Wu L, Xie M, et al. Core-shell structured Fe 304 @TiO 2 -doxorubicin nanoparticles for targeted chemo-sonodynamic therapy of cancer. Int J Pharm 2015;486:380-8.

[35] Wang H, Li M, Hu J, et al. Multiple Targeted Drugs Carrying Biodegradable Membrane Barrier: AntiAdhesion, Hemostasis, and Anti-Infection. Biomacromolecules 2013;14:954-61.

[36] Zhao Z, Yan R, Yi X, et al. Bacteria-Activated Theranostic Nanoprobes against Methicillin-Resistant Staphylococcus aureus Infection. ACS nano 2017;11:4288-4438.

[37] Yang C, Ren C, Zhou J, et al. Dual Fluorescent- and Isotopic-Labelled Self-Assembling Vancomycin for in vivo Imaging of Bacterial Infections. Angewandte Chemie 2017;129:2396-400.

[38] Schroeder A, Avnir Y, Weisman S, et al. Controlling Liposomal Drug Release with Low Frequency Ultrasound: Mechanism and Feasibility. Langmuir the Acs Journal of Surfaces \& Colloids 2007;23:401925 .

[39] Ward M, Wu J, Chiu J. Experimental study of the effects of Optison concentration on sonoporation in vitro. Ultrasound in Medicine \& Biology 2000;26:1169-75.

[40] Lammertink B, Deckers R, Storm G, et al. Duration of ultrasound-mediated enhanced plasma membrane permeability. International Journal of Pharmaceutics 2015;482:92-8.

[41] Yudina A, Lepetit-Coiffé M, Moonen CTW. Evaluation of the Temporal Window for Drug Delivery Following Ultrasound-Mediated Membrane Permeability Enhancement. Molecular Imaging \& Biology Mib the Official Publication of the Academy of Molecular Imaging 2011;13:239-249.

[42] Mitragotri S, Kost J. Low-Frequency Sonophoresis: A Noninvasive Method of Drug Delivery and Diagnostics. Biotechnol Prog 2010;16:488-92.

[43] Su H, Li Z, Dong Y, et al. Damage Effects on Bacille Calmette-Guérin by Low-Frequency, Low-Intensity Ultrasound: A Pilot Study. Journal of Ultrasound in Medicine Official Journal of the American Institute of Ultrasound in Medicine 2016;35:581-587. 
[44] Paris JL, Mannaris C, Cabañas MV, et al. Ultrasound-mediated cavitation-enhanced extravasation of mesoporous silica nanoparticles for controlled-release drug delivery. Chemical Engineering Journal 2018;340:2-8.

[45] Stuart I, Schutt, Esener. Microbubble-mediated ultrasound therapy: a review of its potential in cancer treatment. Drug Design Development \& Therapy 2013;7:375-88.

[46] Chen M, Gan H, Remold HG. A Mechanism of Virulence: Virulent Mycobacterium tuberculosis Strain H37Rv, but Not Attenuated H37Ra, Causes Significant Mitochondrial Inner Membrane Disruption in Macrophages Leading to Necrosis. Journal of Immunology 2006;176:3707-16.

[47] Molloy A, Laochumroonvorapong P, Kaplan G. Apoptosis, but not necrosis, of infected monocytes is coupled with killing of intracellular bacillus Calmette-Guérin. Journal of Experimental Medicine 1994;180:1499-509.

[48] Dallenga T, Repnik U, Corleis Br, et al. M. tuberculosis -Induced Necrosis of Infected Neutrophils Promotes Bacterial Growth Following Phagocytosis by Macrophages. Cell Host \& Microbe 2017;22:519530.

[49] Behar SM, Martin CJ, Booty MG, et al. Apoptosis is an innate defense function of macrophages against Mycobacterium tuberculosis. Mucosal Immunology 2011;4:279-87.

[50] Pang X, Xiao Q, Cheng Y, et al. Bacteria-Responsive Nanoliposomes as Smart Sonotheranostics for Multidrug Resistant Bacterial Infections. ACS nano 2019;13:2427-38.

\section{Figures}

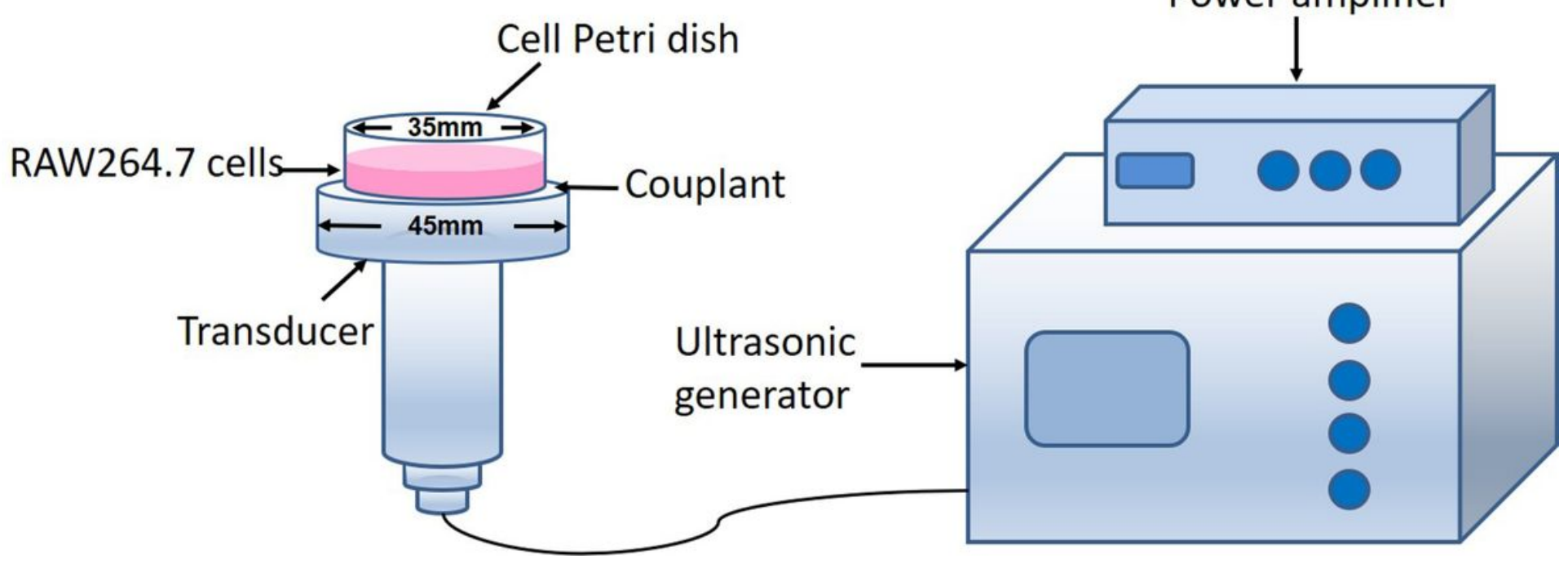

Figure 1 
A schematic diagram of the ultrasonic irradiation procedure. The Cell Petri dish was placed uprightly over the center of the transducer (frequency: $42 \mathrm{KHz}$, diameter: $45 \mathrm{~mm}$ ).

A
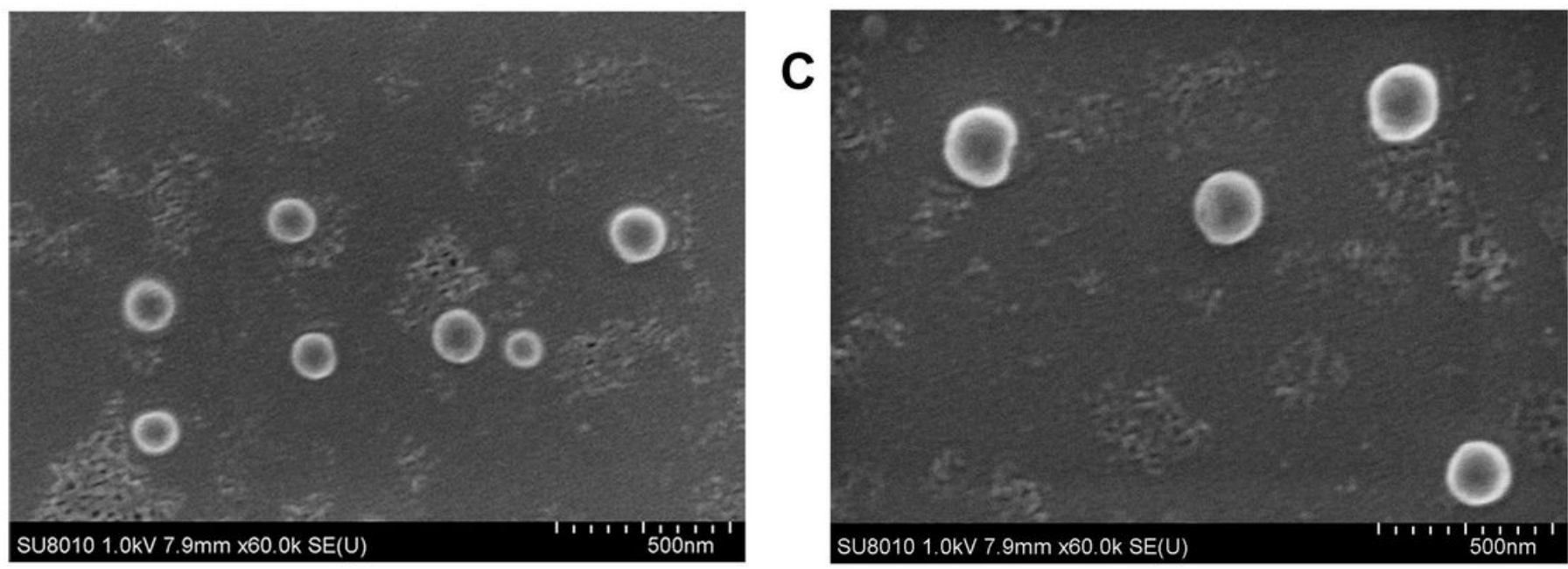

B
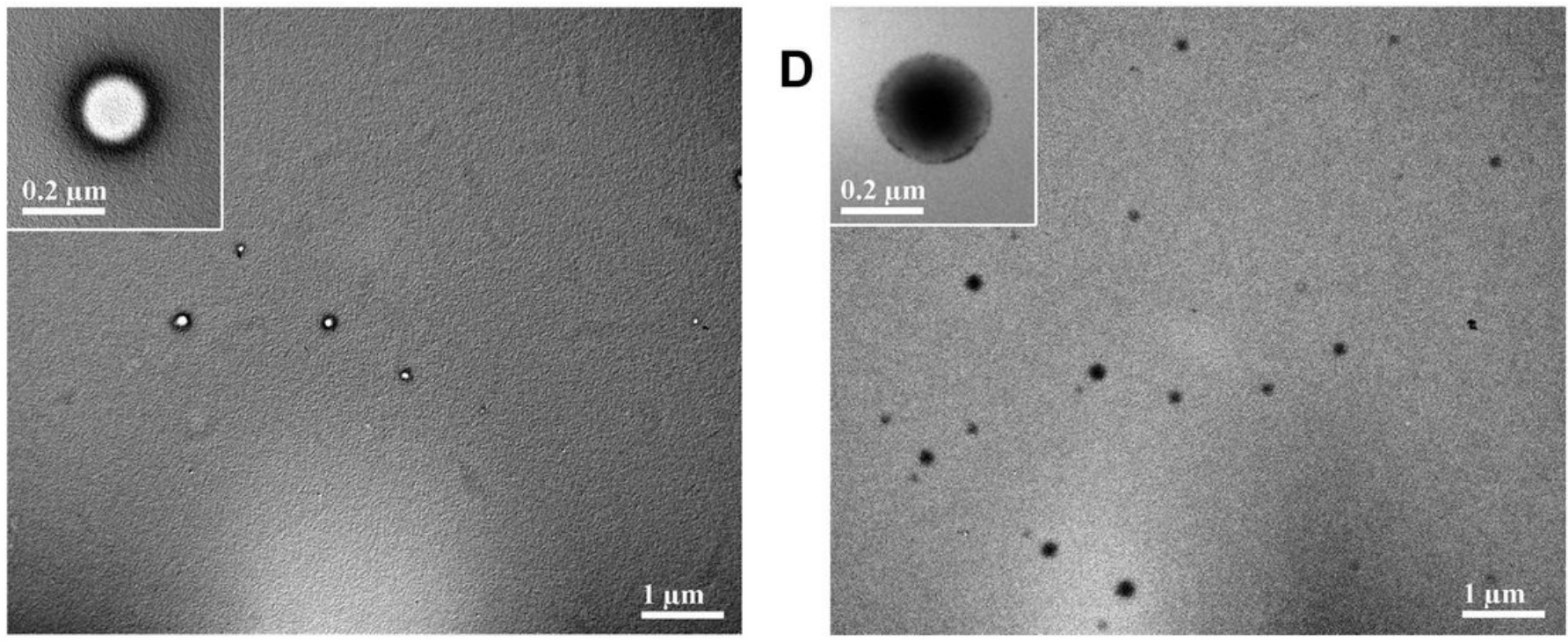

Figure 2

The SEM and TEM images of blank NPs and LEV-NPs. (A) SEM image of blank NPs; (B) TEM image of NPs; (C) SEM image of LEV-NPs; (D) TEM image of LEV-NPs. 


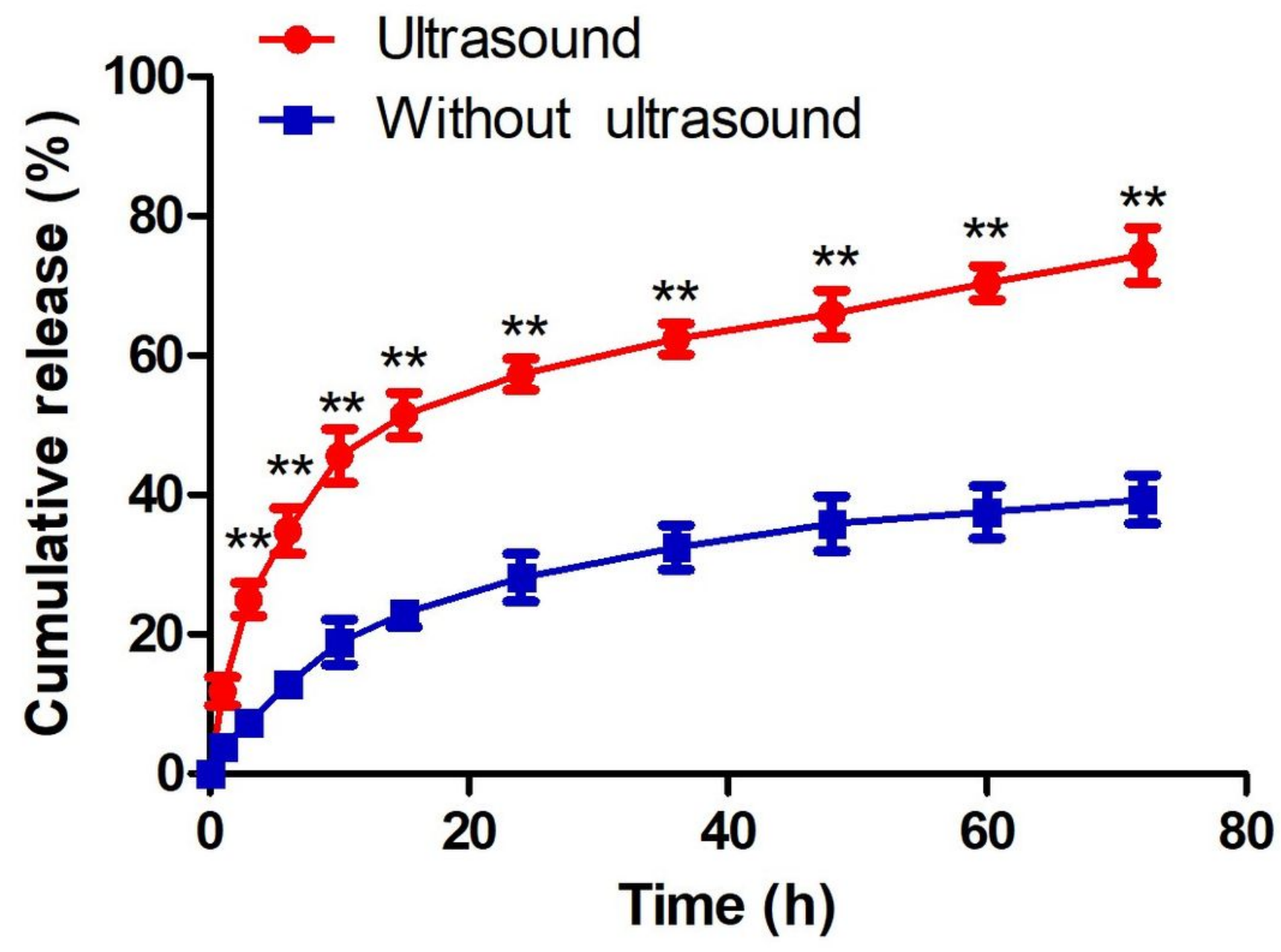

Figure 3

In vitro release profile of LEV from LEV-NPs with ultrasound and without ultrasound treatment. Experiments were performed in triplicates; mean $\pm \mathrm{SD}$ were shown. ${ }^{\star *} \mathrm{P}<0.01$ compared with those without ultrasonic treatment. 


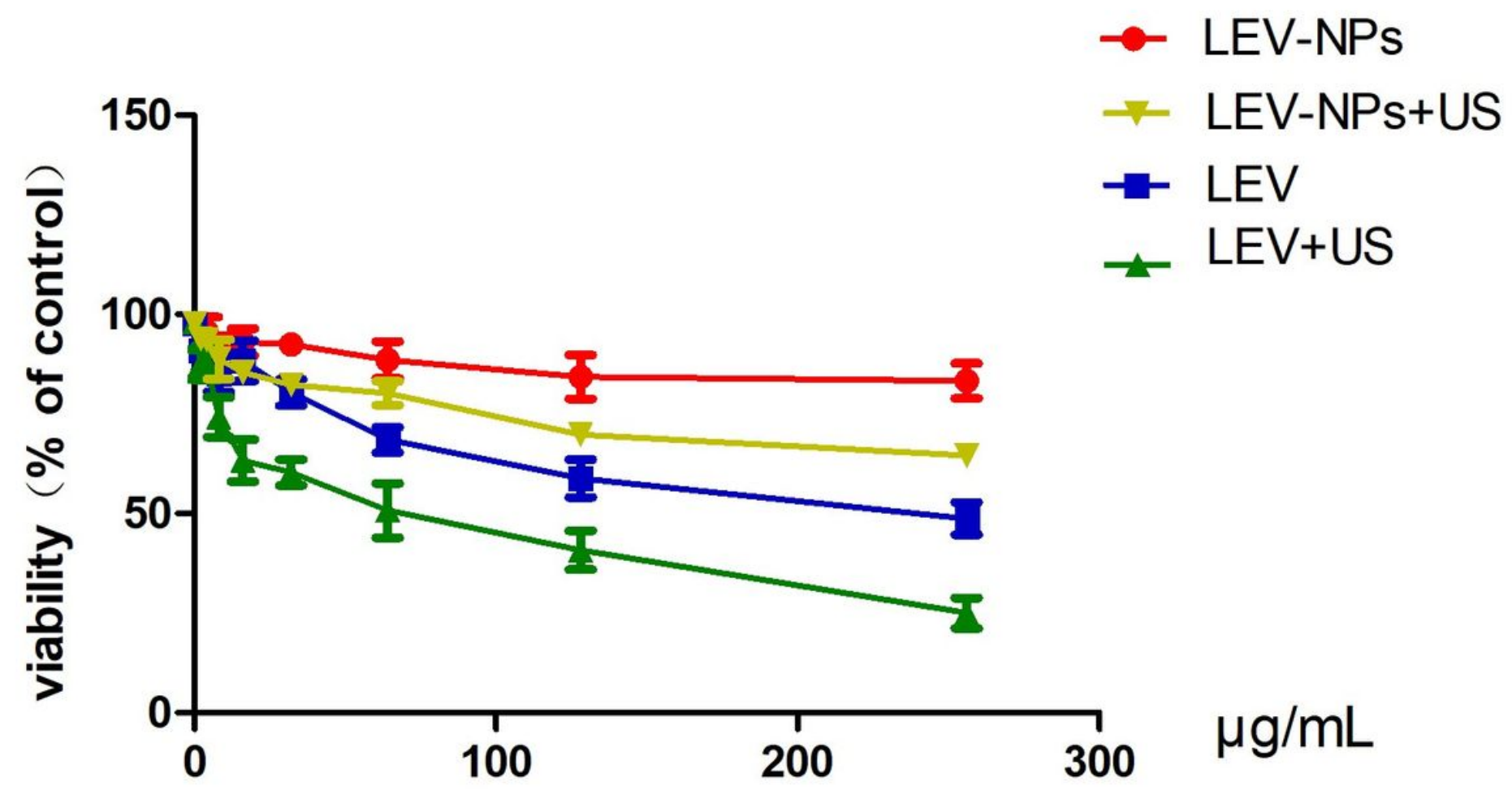

Figure 4

Cytotoxic activity of LEV-NPs at different drug concentrations on RAW264.7 cells. Experiments were performed in triplicates; mean \pm SD were shown. 

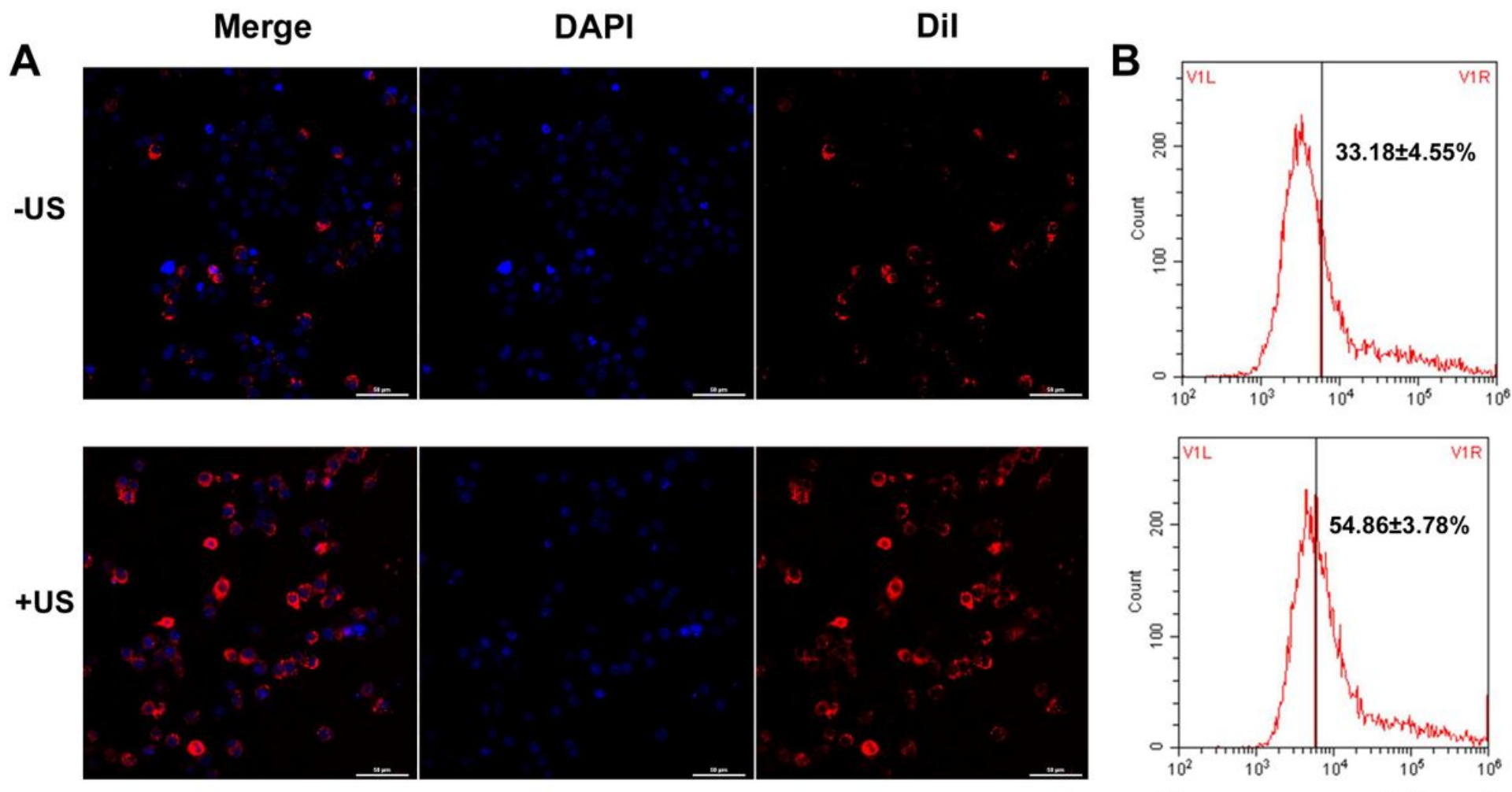

Fluorescence intensity

\section{Figure 5}

Cellular uptake of Dil-NPs by RAW264.7 cells. (A) Intracellular Dil-NPs (red) were observed by laser scanning confocal microscopy, amplification $\times 400$. (B) The fluorescence intensity of intracellular nanoparticles was detected by flow cytometry. Experiments were performed in triplicates; mean $\pm S D$ are shown. $\mathrm{P}<0.05$ when compared the fluorescence intensity of intracellular nanoparticles between US+DilNPs group and Dil-NPs group. 


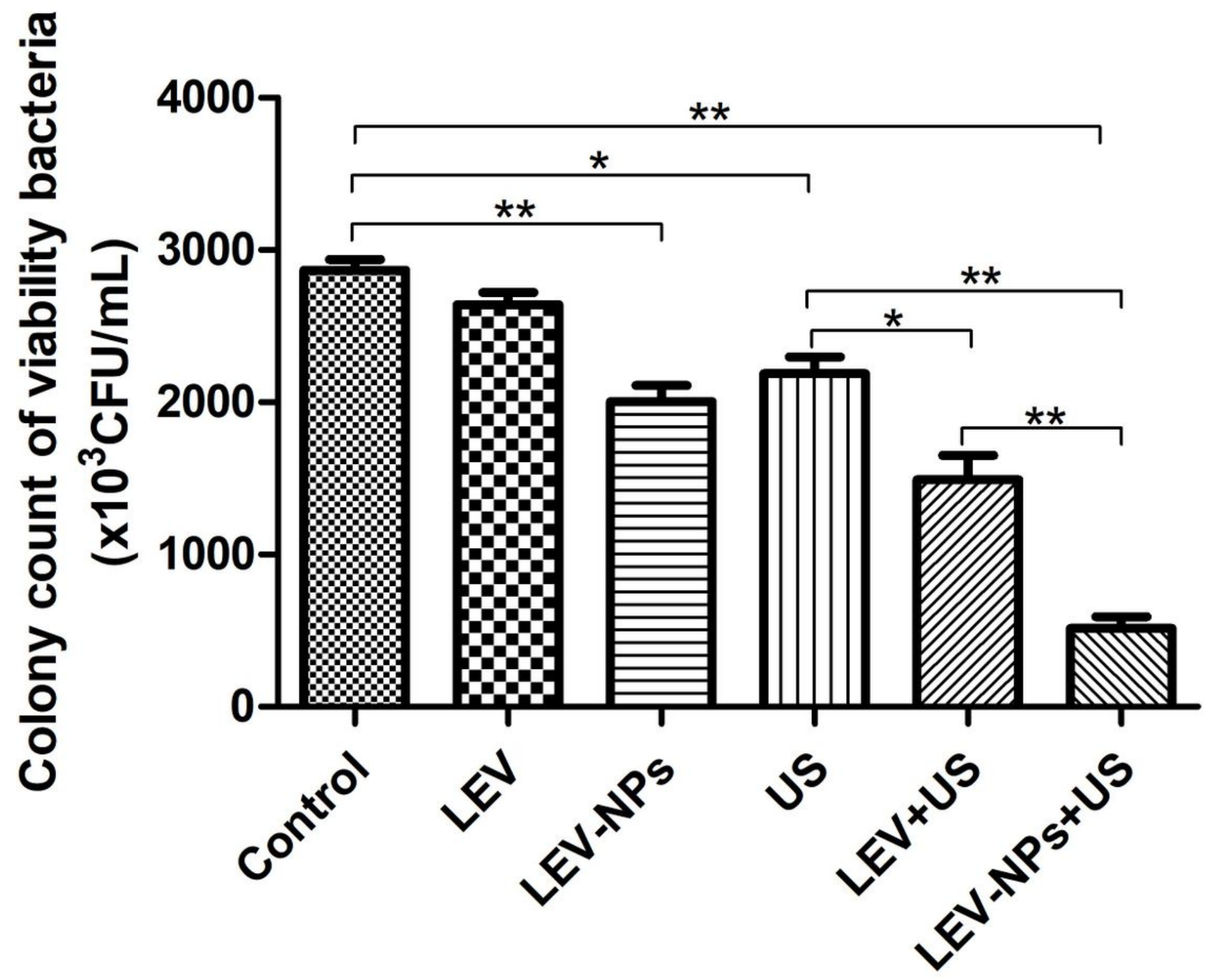

Figure 6

Ultrasound combined with LEV-NPs exhibit intracellular killing activity against M.smegmatis. The bacterial survival rate was determined by CFU assay. The control group did not undergo ultrasound irradiation and drug. Experiments were performed in triplicates; mean $\pm S D$ are shown. ${ }^{*} P<0.05$, ${ }^{*} P<$ 0.01 . 

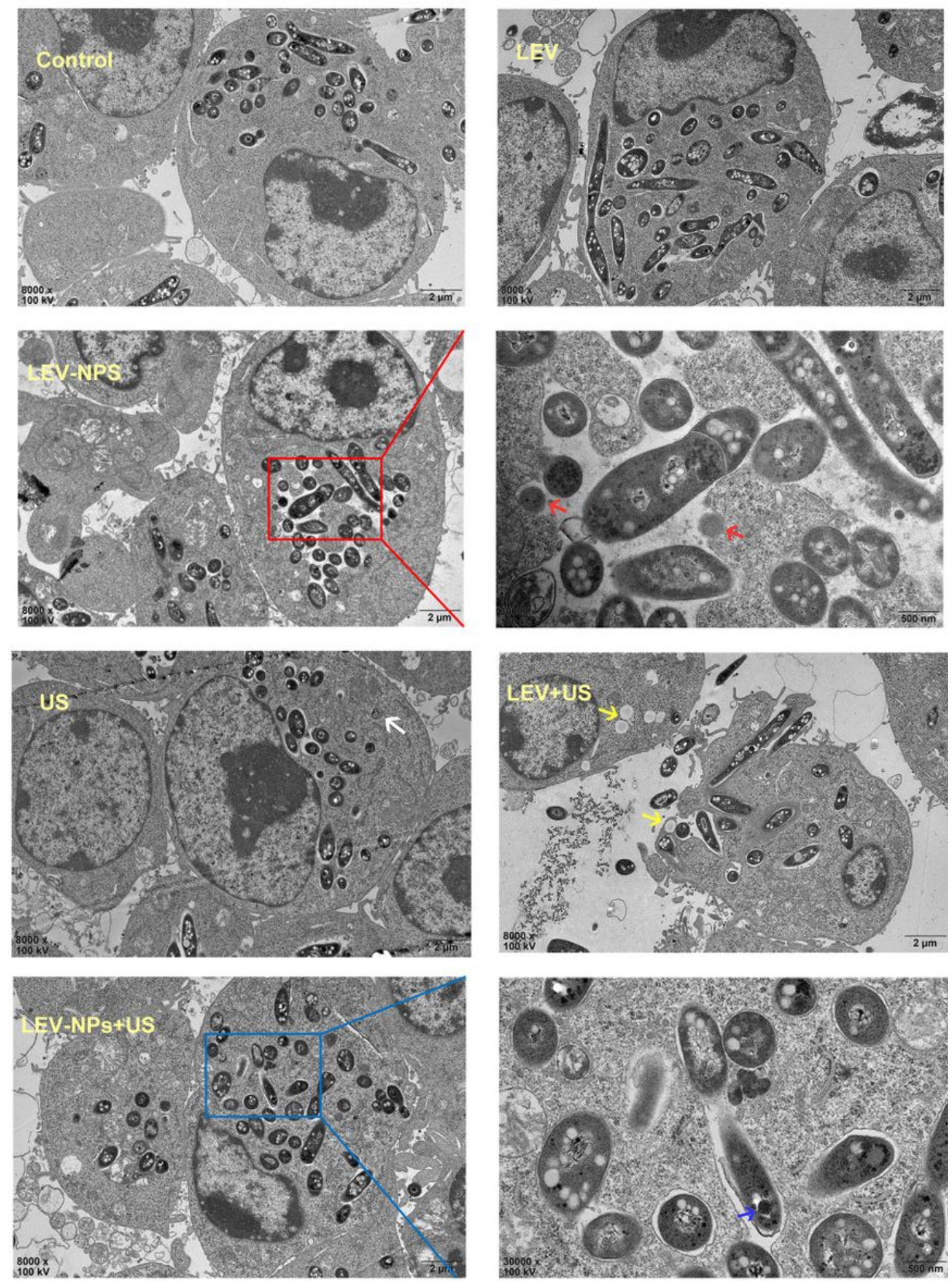

\section{Figure 7}

TEM analyzed the morphological change of RAW264.7 cells and intracellular M. smegmatis in different treatment groups. Intracellular nanoparticles (red arrows) were found in the LEV-NPs group (magnification, $\times 30,000$ ); Nanoparticles (blue arrows) in bacteria were found in the LEV-NPs+US (magnification, $\times 30,000)$. 


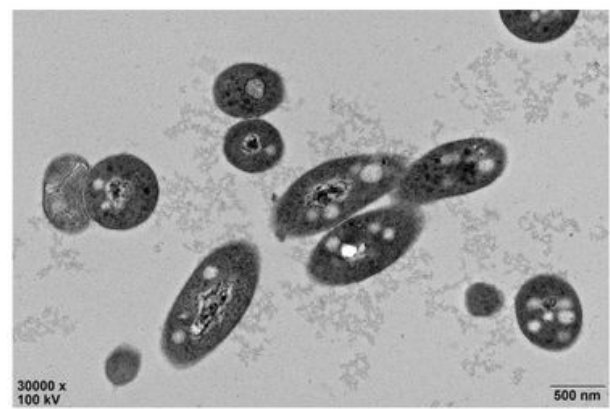

Control

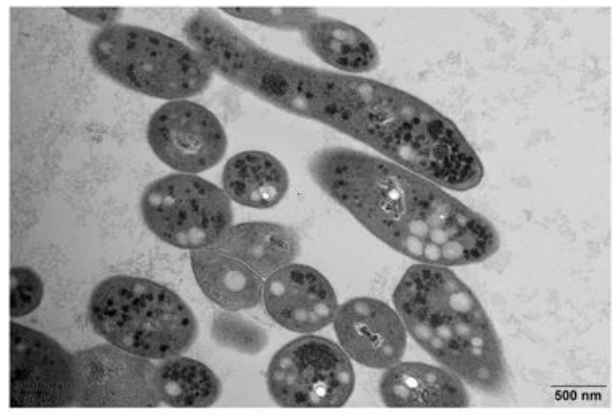

US

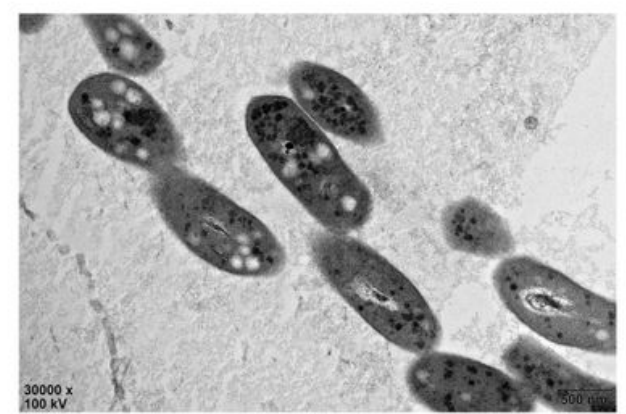

LEV

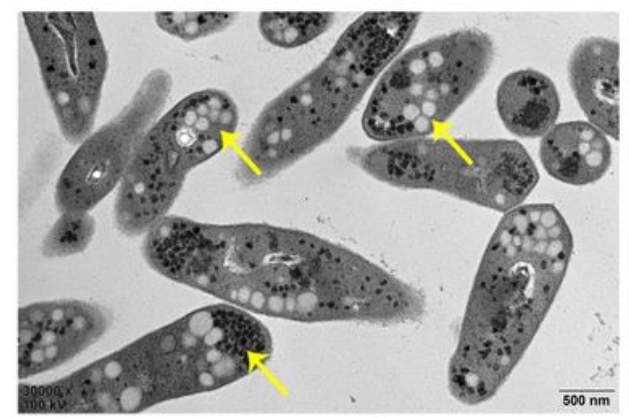

LEV+US

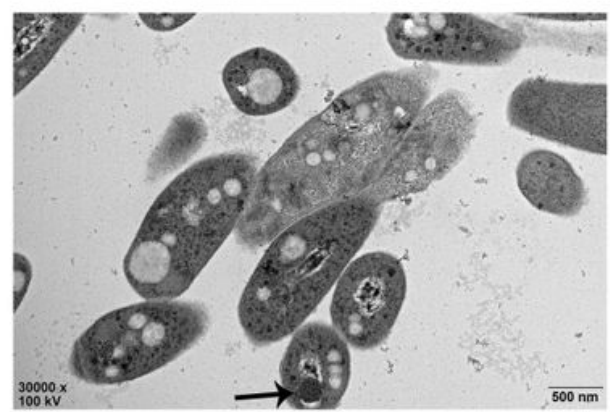

LEV-NPs

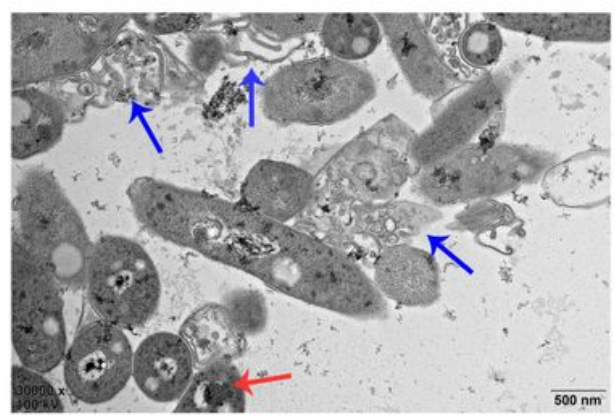

LEV-NPs+US

Figure 8

TEM analyzed the damage of M. smegmatis in different treatment groups. The black arrow indicates LEV-NPs; The yellow arrow indicates lipid droplet and glycogen; The blue arrow indicates the broken cell wall, and the red arrow indicates LEV-NPs.(magnification, $\times 30,000$ ) 


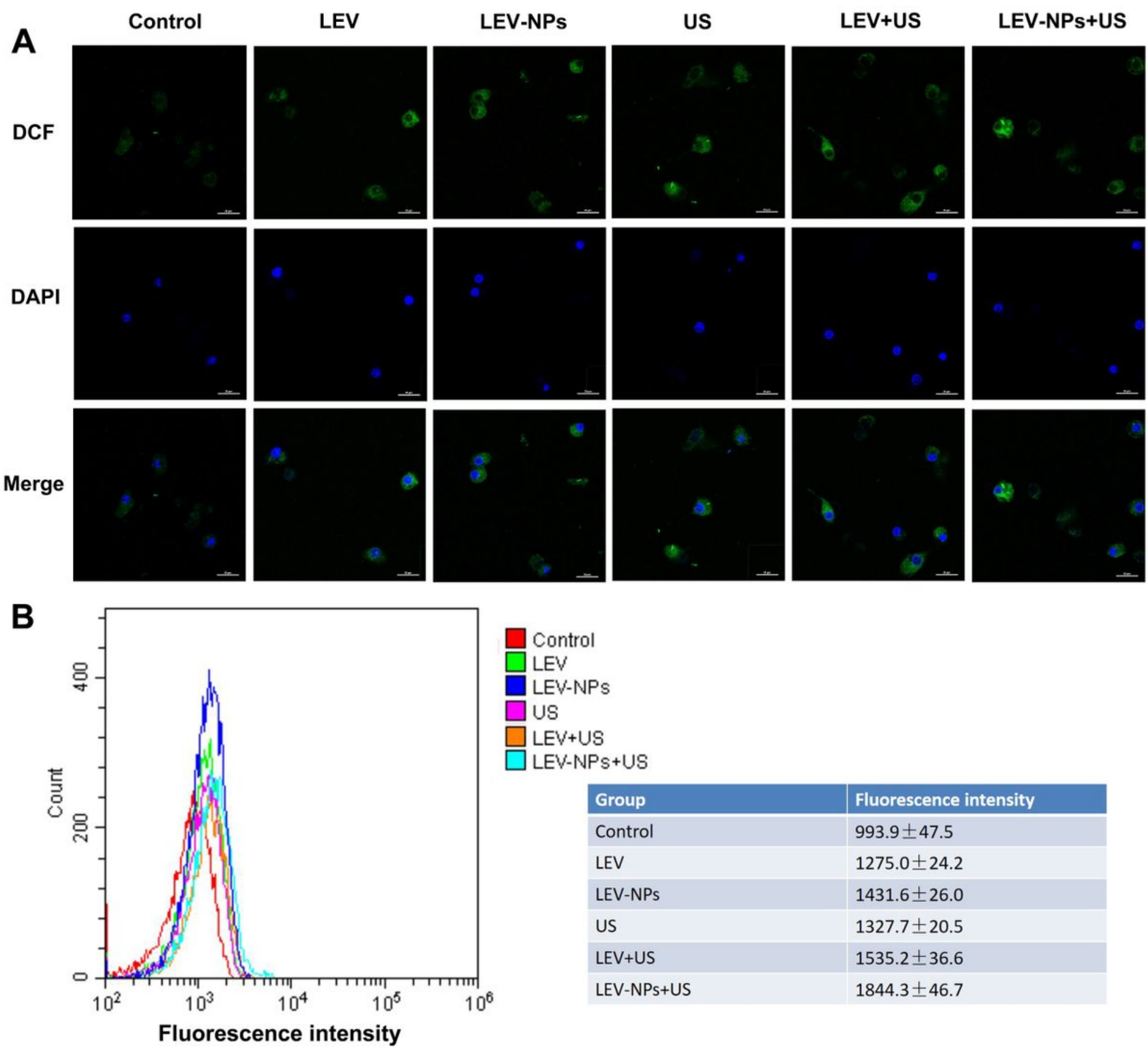

Figure 9

Intracellular ROS production determination. (A) The intracellular ROS (green) of different treatment were detected by laser confocal microscopy, amplification $\times 400$. (B) The intracellular ROS level in different treatment groups was analyzed by flow cytometry. Experiments were performed in triplicates; mean \pm SD are shown. $\mathrm{P}<0.05$ compared with control. 

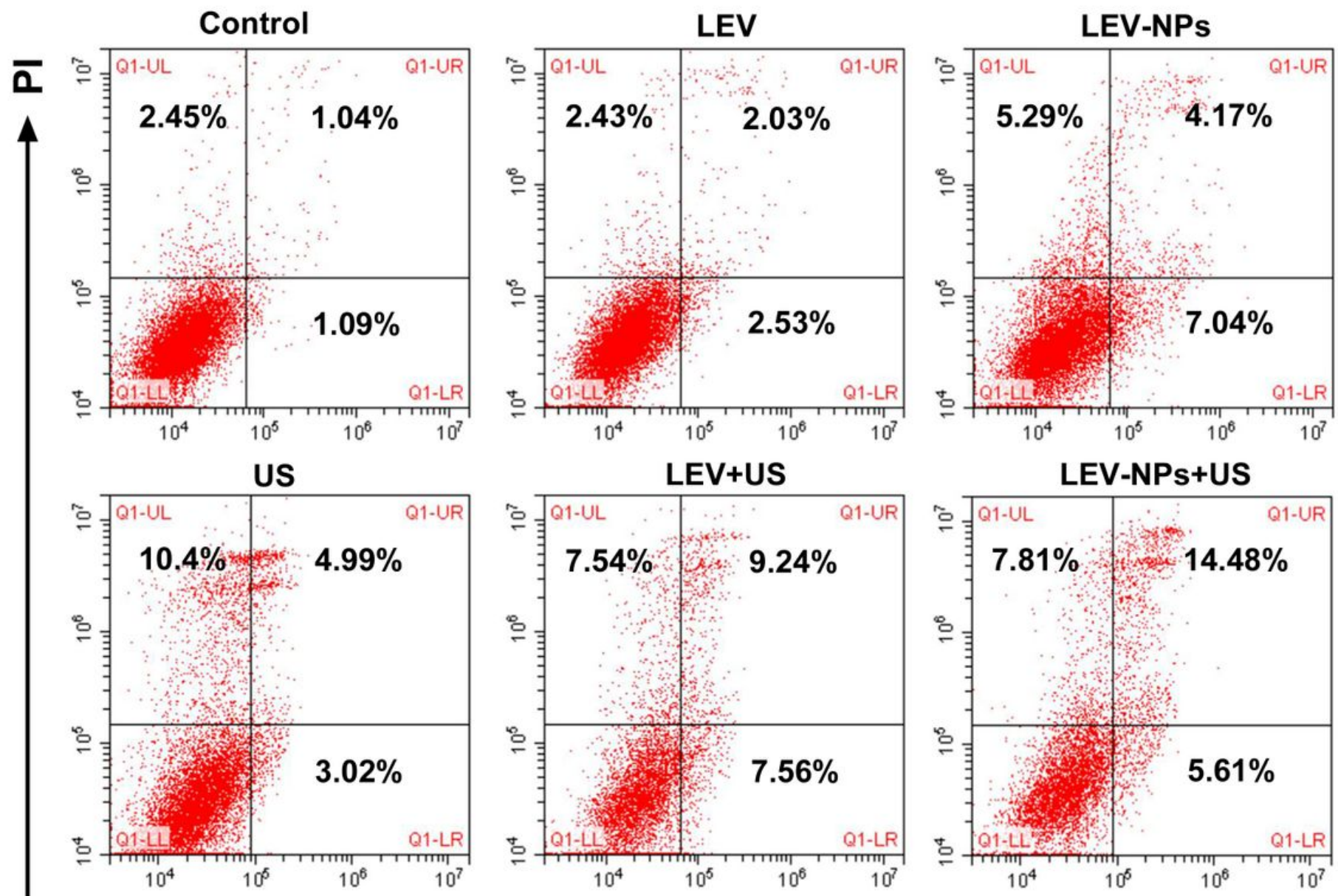

Annexin-V

Figure 10

Apoptosis and necrosis ratio of RAW264.7 cells determined by flow cytometry after double staining with Annexin-V/PI. The upper right quadrant indicated early apoptotic cells, the lower right quadrant indicated late apoptotic cells, the upper left quadrant indicated necrotic cells, and the lower-left quadrant indicated normal cells. 